\title{
On the construction of the cerebral hemodynamics model based on clinical data
}

\author{
A.A. Cherevko ${ }^{1,4 *}$, M.A. Shishlenin ${ }^{2,3,4}$, A.K. Khe ${ }^{1,4}$, E.E. Bord ${ }^{4}$, V.V. Berestov ${ }^{5}$, \\ K.Y. Orlov 5 , V.A. Panarin ${ }^{5}$ \\ ${ }^{1}$ Lavrentyev Institute of Hydrodynamics of SB RAS, Novosibirsk, Russia \\ ${ }^{2}$ Sobolev Institute of Mathematics of SB RAS, Novosibirsk, Russia \\ ${ }^{3}$ Institute of Computational Mathematics and Mathematical Geophysics, Novosibirsk, Russia \\ ${ }^{4}$ Novosibirsk State University, Novosibirsk, Russia \\ ${ }^{5}$ Meshalkin national medical research center, Novosibirsk, Russia \\ *e-mail: cherevko@mail.ru
}

Key words: hemodynamics, neurosurgery, arterial aneurism, nonlinear oscillator, inverse problem, gradient method

Currently, the monitoring of the hemodynamics of the brain is being implemented by neurosurgeons of the National Medical Research Center of Academician E. Meshalkin in collaboration with colleagues from the Lavrentyev Institute of Hydrodynamics.

This material, which is unique in the world practice, made it possible to approach the construction of a mathematical model of hemodynamics.

As the model, the nonlinear oscillator equation was chosen. In this equation, the velocity is a "governance" function (the right-hand side of the equation), and the second-order differential operator acting on the pressure. The "blood flow - vessel - brain substance" system is nonlinear and has both elastic and damping properties, for this reason the model of generalized Van der Pol-Duffing equation was suggested to identify the characteristic behavior of hemodynamic parameters in the surroundings of vascular pathologies. Equation coefficients characterize individual living system of the patient, the measurement location, the presence of anomalies. The coefficients of this equation are individual for each patient.

We solve coefficient inverse problem to determine the coefficients of this model by known clinical intraoperational data. This model adequately describes the behaviour of hemodynamic parameters.

We investigate and construct numerical method for solving the coefficient inverse problem for essentially nonlinear ODE by clinical data of neurosurgical operation. We recover the coefficients by clinical data obtained during neurosurgical operation in vicinity of arterial aneurysm, that a pathological enlargement (dilation) of the artery.

The proposed model and the method for solving the inverse problem together allowed us to restore the behavior of pressure in the vicinity of intracranial vascular pathology, having data on the blood flow velocity in the "real" time. Investigation of the dependence of pressure on velocity in blood vessels is of great practical importance, since there are currently non-invasive methods for measuring speed (tomography, ultrasound), but no non-invasive methods of measuring pressure. At the same time, information about pressure is important.

We study the relationship between the properties of this equation and the state of the vascular bed.

Acknowledgements: Supported by RFBR (projects No. 17-08-01736) and MSC RK grant AP05134121. 